\title{
Bi-modal Structure of Copper via Room-Temperature Partial Recrystallization After Cryogenic Dynamic Compression
}

\author{
DONG-HYUN AHN, DONG JUN LEE, MINJU KANG, LEE JU PARK, \\ SUNGHAK LEE, and HYOUNG SEOP KIM
}

PURE copper was compressed at high strain rates (over $\sim 3 \times 10^{3} \mathrm{~s}^{-1}$ ) under liquid nitrogen. This deformation resulted in bi-modal microstructures of ultrafine grains and abnormally grown micro grains, and in greater hardness (by $\sim 30 \mathrm{Hv}$ ) than room-temperature, dynamically deformed copper. This bi-modal microstructure is attributable to partial recrystallization at room temperature, activated by high-energy states and by twins generated at high ZenerHollomon parameter conditions. This result demonstrates a new approach for producing bi-modally structured materials.

DOI: $10.1007 / \mathrm{s} 11661-016-3326-9$

(C) The Minerals, Metals \& Materials Society and ASM International 2016

\section{INTRODUCTION}

THE control of microstructure as a means to improve useful properties is an everlasting issue in material science and engineering. Recently, extreme processing approaches, such as large strain, high strain rates, and low temperature, have attracted much interest for designing microstructure. In particular, severe plastic deformation (SPD), by which giant strain deformations are applied in order to obtain ultrafine/nano microstructures, has been vigorously researched over the past two decades. ${ }^{[1-5]}$ For example, ultrafine/nano materials of very high strength can be fabricated via high-pressure torsion (HPT) or equal-channel angular pressing (ECAP). However, these SPD processes have limitations for decreasing grain size and controlling microstructure. In order to further decrease the lower bound of the grain size, and to expand the microstructural window of SPD-processed materials, many researchers have been interested in controlling processing parameters, such as strain rate and temperature, at extreme levels. ${ }^{[6-10]}$

The simultaneous effects of strain rate and temperature on microstructural evolution during plastic deformation are generally expressed by the Zener-Hollomon parameter, $Z$, as follows:

DONG-HYUN AHN and MINJU KANG, Ph.D. Candidates, and SUNGHAK LEE and HYOUNG SEOP KIM, Professors, are with the Department of Materials Science and Engineering, POSTECH (Pohang University of Science and Technology), Pohang 790-784, Korea. Contact e-mail: hskim@postech.ac.kr DONG JUN LEE, Senior Researcher, formerly with the Materials Deformation Department, Korea Institute of Materials Science (KIMS), Changwon 641831 , Korea, is now with the Commercialization Research Division, Korea Institute of Material Science (KIMS), Changwon, Korea. LEE JU PARK, Principal Researcher, is with the Agency for Defense Development (ADD), Daejeon 305-152, Korea.

Manuscript submitted March 5, 2015.

Article published online January 20, 2016

$$
\text { In } Z=\text { In } \dot{\varepsilon}+\frac{Q}{\mathrm{RT}},
$$

where $\dot{\varepsilon}, Q, R$, and $T$ are strain rate, activation energy for diffusion, the universal gas constant, and temperature, respectively. In general, as the deformation condition reaches extreme levels, such as high speed and low temperature, the $Z$ value increases, indicating high strength and refined microstructure. To understand the effect of the $Z$ parameter at not only extreme but also ordinary conditions, the deformation of copper over a wide range of temperature and strain rate were studied. ${ }^{[6-8]}$ However, these studies have three deficiencies: (1) few study at strain rates over $\sim 10^{3} \mathrm{~s}^{-1}$ and at cryogenic temperature, (2) less consideration for global microstructural features than for local microstructural ones, and (3) insufficient attention to reheating from cryogenic temperature to room temperature after the deformation.

In this study, the effects of extremely high strain rate and low temperature on the evolution of microstructure were investigated by performing cryogenic dynamic compression of copper. In particular, both local and global microstructures were analyzed, considering their deformation and recovery environments.

\section{EXPERIMENTAL PROCEDURE}

Cylindrical samples (4.0 mm height, $4.0 \mathrm{~mm}$ diameter) of commercial pure (99.97 pct) copper (Figure 1(a)) were prepared for successive compression at high strain rates at room temperature $\left[\sim 298 \mathrm{~K}\left(\sim 25^{\circ} \mathrm{C}\right)\right]$ and at the temperature of liquid nitrogen $(\sim 77 \mathrm{~K}$ $\left.\left(-196{ }^{\circ} \mathrm{C}\right)\right)$.

Six stopper rings of different heights $(3.7,2.7,2.0$, $1.5,1.1$, and $0.7 \mathrm{~mm}$ ) were prepared. Four of them had outer diameters of $19.0 \mathrm{~mm}$ and inner diameters of $10.0 \mathrm{~mm}$, but the rings of 1.1 and $0.7 \mathrm{~mm}$ height had 


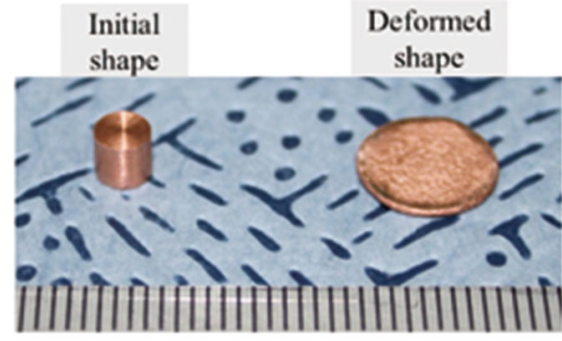

(a)

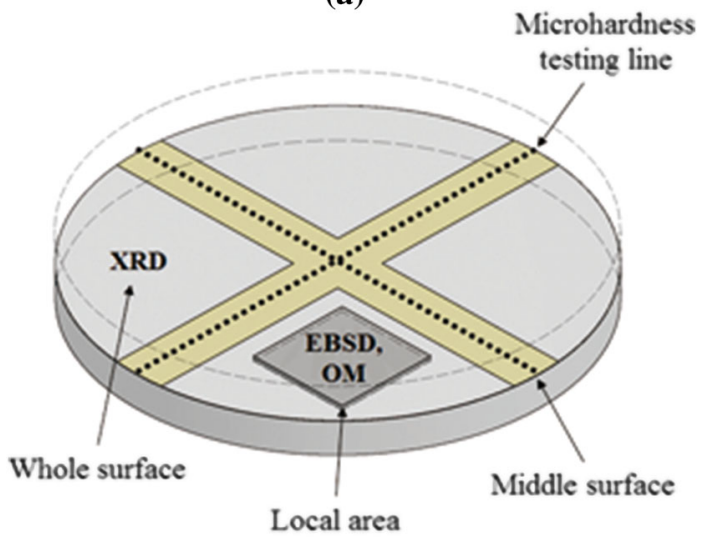

(b)

Fig. 1-(a) Initial and deformed sample at high strain rate using split Hopkinson pressure bar system, (b) schematic diagram of the deformed sample with marking where micro-hardness, XRD, and EBSD have been measured.

inner diameters of 12.0 and $14.0 \mathrm{~mm}$, respectively. The stopper rings were needed to control the generation of adiabatic heat during the dynamic deformations. The dynamic compressions were performed using a split Hopkinson pressure bar (SHPB) system, which is widely used for achieving high-strain-rate compression (over $10^{3} \mathrm{~s}^{-1}$ ). With this system, the levels of deformation were adjustable (six steps using the stopper rings). Each deformation was implemented at an average strain rate of $\sim 3 \times 10^{3} \mathrm{~s}^{-1}$. Two samples were deformed up to a true strain of 1.74 (from initial 4.0 $\mathrm{mm}$ to final $0.7 \mathrm{~mm}$ heights) at room temperature (hereafter, RTD sample: room-temperature-deformed sample) or at cryogenic temperature by immersing the samples in liquid nitrogen during deformation (CTD sample: cryogenic-temperature-deformed sample). The temperature increment $\Delta T$ during the adiabatic deformation may be calculated using the following energy conservation equation:

$$
\Delta T=\frac{\alpha}{C \rho} \int \sigma d \varepsilon
$$

where $\alpha, C, \rho, \sigma$, and $\mathrm{d} \varepsilon$ are the fraction of plastic work converted into heat, the specific heat, the density of copper, the flow stress, and the strain increment, respectively. The parameter $\alpha$ was assumed to be 0.9 , and temperature-dependent specific heat $C$ was applied. ${ }^{[11,12]}$ A maximum constant flow stress $\sigma$ of $700 \mathrm{MPa},{ }^{[13]}$ and average increment of strain $\mathrm{d} \varepsilon$ of $\sim 0.3$ were chosen at each deformation step. The calculated temperature increment $\Delta T$ was $\sim 80 \mathrm{~K}$.
After the compression, the surfaces perpendicular to the direction of deformation were polished using silicon carbide paper (300-1200 grit) and diamond suspensions (3 and $1 \mu \mathrm{m}$ ) until the middle plane of the specimen appeared. The selection of the plane instead of the plane parallel to the direction was made in order to secure the largest area for more representative X-ray diffraction (XRD) measurements with high intensity.

A synchrotron XRD measurement was performed using the 9B beam line at the Pohang Light Source (PLS, Pohang, South Korea, for more details about the system visit: http://pal.postech.ac.kr/paleng/bl/9B/), with a resolution of $\Delta E / E=\sim 2 \times 10^{-4}$ filtered by a double crystal monochromator using a Si (111) crystal. The beam had $0.1497 \mathrm{~nm}$ of the calibrated wavelength and the beam size was $1 \mathrm{~mm} \times 20 \mathrm{~mm}$ and the sample holder was continuously rotating, which enables X-rays to cover the whole surface, as shown in Figure 1(b). The surface of the sample was placed on the aligned position at which beam was reflected into the detector. The measured range of two theta was set from 40 to 120 with 0.01 step size per 3 seconds.

The micro-hardness of the samples was measured on polished surfaces using a Vickers hardness tester (FM-700, Future-Tech. Corp., Japan) with $300 \mathrm{~g}$ load and 10 seconds dwell-time along the black-dotted lines shown in Figure 1(b).

The deformed surface microstructures marked in Figure 1(b) were observed using optical microscopy (OM, Olympus BX51M). Before observations were made, the surface was etched using colloidal silica during polishing the surface. Electron backscattered diffraction (EBSD) measurements were performed on the same surfaces as used for the OM observation, using the focused ion beam/EBSD system (Helios, Pegasus, FEI). The observed plane was mechanically polished and then electro-polished using an electric polisher (LectroPol-5, Struers), with D2 solution and $24 \mathrm{~V}$ at room temperature.

\section{RESULTS AND DISCUSSION}

Figure 1(a) exhibits the samples before $(4.0 \mathrm{~mm}$ height, $4.0 \mathrm{~mm}$ diameter $)$ and after $(0.7 \mathrm{~mm}$ height, 9.5 $\mathrm{mm}$ diameter) the SHPB compression. Figure 2 is a plot of the distribution of Vickers hardness of the RTD and CTD samples. From the hardness distribution and OM images from center to edge, global differences in mechanical property and microstructure were not found. The RTD sample had high micro-hardness ( $>140 \mathrm{Hv}$, compared to the initial hardness of $\sim 45 \mathrm{Hv}$ ) across the sample. This hardness level $(\sim 140 \mathrm{Hv})$ is a well-known value of the saturated hardness after SPD of copper at room temperature. ${ }^{[10]}$ The CTD sample exhibited a much greater hardness $(\sim 170 \mathrm{Hv}$, more than $\sim 30 \mathrm{Hv})$ than did the RTD sample $(\sim 145 \mathrm{Hv})$. Such an increase in hardness in the low-temperature compressed samples could be attributed to a suppression of defect recovery and to smaller grains. ${ }^{[14]}$ For example, lower temperature hinders the recovery of dislocations. This indicates that higher dislocation densities remained after 
deformation. By the principle of similitude, higher dislocation density indicates finer grain size as a result of the characteristic wavelength of the dislocation patterns. ${ }^{[15]}$ Although the total strain of $\sim 1.74$ is generally not enough for a saturation of hardness evolution by SPD, low temperature and high strain rates enable the saturation of hardness to be advanced, even at this moderate strain level. The difference in hardness between the RTD and CTD samples is similar to the difference in saturated hardness of HPT copper processed at room and at cryogenic temperatures. ${ }^{[10}$

The OM microstructures of the RTD and CTD samples are shown in Figures 3(a) and (b), respectively. Two upper images in Figures 3(a) and (b) were obtained at low magnification and the others from high-magnification observation. It is difficult to resolve individual grains with OM due to severe deformations in both samples; however, some white regions are noticeable (marked by red arrows). In higher magnification images, there are two clearly different regions in both samples: white and gray regions.

Although both samples exhibited similar microstructures, two distinguishing features were found: the direction of etching traces and the degree of clearance

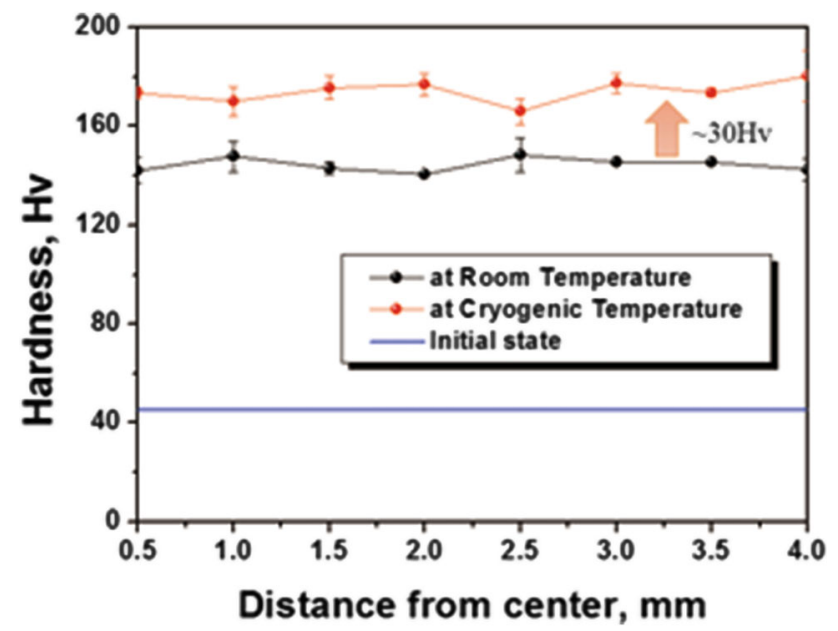

Fig. 2-Micro-hardness distributions along the diameter of the sample.
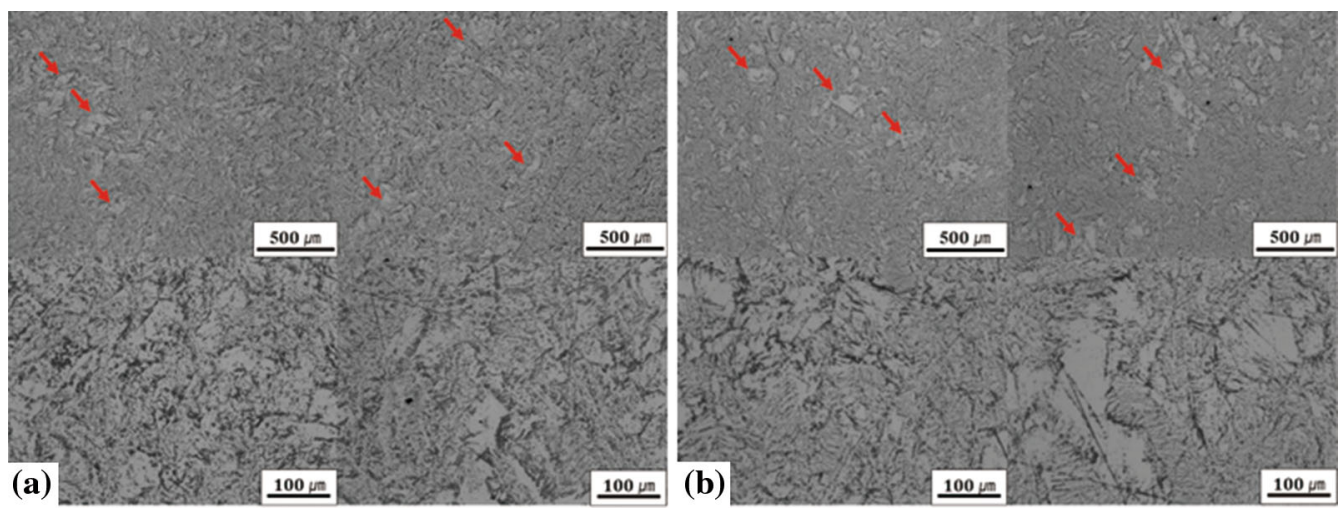

in the white regions. First, in the RTD sample, the etched phase-boundaries traces had random and complicated shapes; in contrast, the etched traces in some gray regions of CTD sample were aligned which may be associated with deformation twin. ${ }^{[9]}$ It has been reported that deformation twinning occurs more easily during cryogenic dynamic deformation than during room-temperature or quasi-static deformation. ${ }^{[8]}$ Figures 4 (a) and (b) show the evidence of deformation twinning (marked by arrows) at intermediate deformation stage having strains $\sim 0.3$ and $\sim 0.65$, respectively.

Second, the white regions in the RTD sample include several etched spots or lines, and unclear region-boundaries, whereas relatively clear etching areas and region-boundaries are found in the CTD sample. The difference between white and gray regions could be attributed to the difference in the feature of microstructure. That is, different microstructural evolutions introduced different etching trends in the same specimen, like hard-to-etch (white) and easy-to-etch (gray) regions. ${ }^{[9]}$ However, the clear difference in etched features indicates only that there exist different microstructure histories causing strong inhomogeneity in CTD sample. For more specific characterizations, other concrete evidence, such as EBSD and XRD results, should be supported.

The similar features were also observed in the EBSD results. The top images in Figure 5 present inverse pole figure (IPF), and the bottom images show grain orientation spread (GOS) maps of (a) the initial sample, (b) RTD sample, and (c) and (d) CTD samples. Distinct undeformed features were found in the initial sample, which had a coarse average grain size of $\sim 30 \mu \mathrm{m}$. After the dynamic deformation at room temperature, the microstructure was highly refined to sub-micron scale. Although some coarse grains were not perfectly refined to form sub-micron grains surrounded by high-angle grain boundaries (HAGBs), the grains were divided by low-angle grain boundaries (LAGBs) showing orientation gradients in grains. As seen in the OM images, there is inhomogeneity in the microstructure of the RTD and CTD samples. Such inhomogeneous features showing some less refined regions (coarser grains than periphery grains) were also found in the EBSD images (marked by the arrows in the RTD sample, CTD sample 1, and

Fig. 3-Optical microscope images of the samples deformed (a) at room temperature and $(b)$ at cryogenic temperature. 


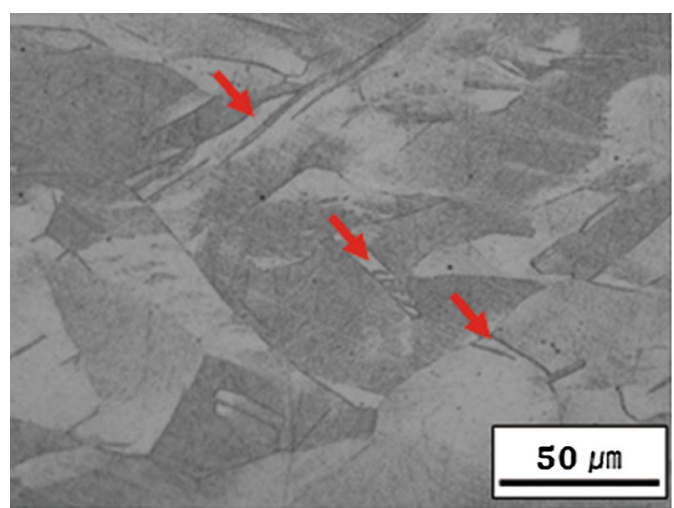

(a)

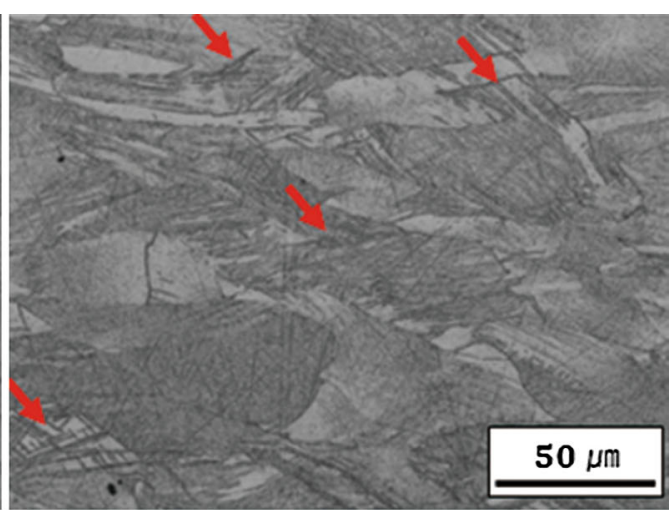

(b)

Fig. 4 Optical microscope images of the samples deformed at strain $(a) \sim 0.3$ and $(b) \sim 0.65$ in CTD sample. Arrows point the traces of deformation twinning.

CTD sample 2). In the GOS map of the RTD sample, the areas of coarse grain are actually highly deformed areas with dislocation cell substructures, and they lack HAGBs. It should be noted that dislocation cell structures and LAGBs are less etchable than HAGBs. ${ }^{[9]}$ More refined microstructure was observed in the IPF maps of the CTD sample than in the RTD sample. The alignment of the refined microstructure, as seen in the OM images, was also observed (the dotted circle region in the IPF map of the CTD sample 1). Twinning in the CTD sample can be confirmed from the shape and boundary misorientations. The grains coarser than the surrounding grains were also found in the CTD sample 1 and CTD sample 2; however, their microstructural information is clearly different. Although the characteristics of the coarse grains in CTD sample 1 were similar to those of the RTD sample, the coarse grains in the CTD sample 2 had very low GOS values. This low range indicates recrystallized or initially undeformed-state grains.

From the microstructural results, it can be concluded that the CTD sample underwent local recrystallization, and, as a result, a bi-modal structure (sub-micron and micron grain sizes) was generated. In order to support this conclusion, the XRD patterns were compared in Figure 6. Since the patterns had very low background noise around $\sim 200$ and maximum intensity over $\sim 28,000$ in CTD sample, no background subtraction was applied. In Figure 6(a), all the peaks in the RTD and CTD samples were pure copper peaks. Plastic deformations generate defects and refine the microstructure; hence, the XRD peaks were broadened. Figure 6(b) shows the reference peak from the standard LaB6 sample, with few defects and large crystallite size, and the (200) peaks from the RTD sample and CTD sample in logarithmic scale. When a sample is deformed, the whole peak from bottom to top is broadened, as seen for the RTD sample. Interestingly, the top part of the peak for the CTD sample presents a shrunk sharp shape. This feature is also found in other peaks of the CTD sample, e.g., (111), (220), and (311), in Figure 6(c). From the results of the microstructural inhomogeneity in the OM and EBSD analyses, and the simple superposition of the sharp peaks (defect-free, micro-grain size) and broadened peaks (many defects, sub-micron grain size), it can be concluded that bi-modal microstructure, i.e., a combination of the sub-micron scale and severely deformed grains, and the micron scale and fewer defect grains, developed in the CTD sample (Figure 6(d)).

There are two explanations for the coarse grains that appeared in the ultrafine-structured CTD sample: in situ grain growth during deformations ${ }^{[16,17]}$ and recrystallization. ${ }^{[18]}$ The former (grain growth) is attributed to non-uniform grain boundary mobility of ultrafine materials, which leads to sub-grain growth and local inhomogeneous distribution of stress, and to subsequent grain rotation for sub-grain agglomeration. Although grain growth could occur through this process, defects were not released and the grain size was not greater than micron scale. ${ }^{[16]}$ Therefore, the strain-free microstructure and grain size greater than a micron in the CTD sample indicate that the former reason is not plausible.

In the latter (recrystallization) case, two types of recrystallization exist: one induced by rotation and one induced by nucleation and growth. ${ }^{[18,19]}$ Recrystallization by rotation is frequently called dynamic recrystallization (in situ or continuous recrystallization) and is a common phenomenon in metals that are greatly deformed. ${ }^{[20,21]}$ The grain size generated by dynamic recrystallization is normally dependent on the strain rate and temperature; that is, smaller grains are generated at lower temperature or under higher strain. Such recrystallized grains are observed in the EBSD results of both the RTD and CTD samples, where the recrystallized, defect-free grains are defined by the blue region on the GOS map. Comparing the RTD and CTD samples, different recrystallized grain sizes are found: the CTD sample has smaller defect-free grains except for some very large defect-free grains. The size of these exceptional grains confirms that they did not originate via dynamic recrystallization.

Actually, the formation mechanism of the bi-modal structure of the CTD sample is similar to that of the SPD-processed and annealed samples. ${ }^{[22-26]}$ There is a range of temperature and required time for partial recrystallization induced by nucleation and growth (called migration recrystallization).$^{[19]}$ This type of recrystallization is well known even in conventional-SPD-processed materials. 
Inverse Pole Figure (IPF) maps
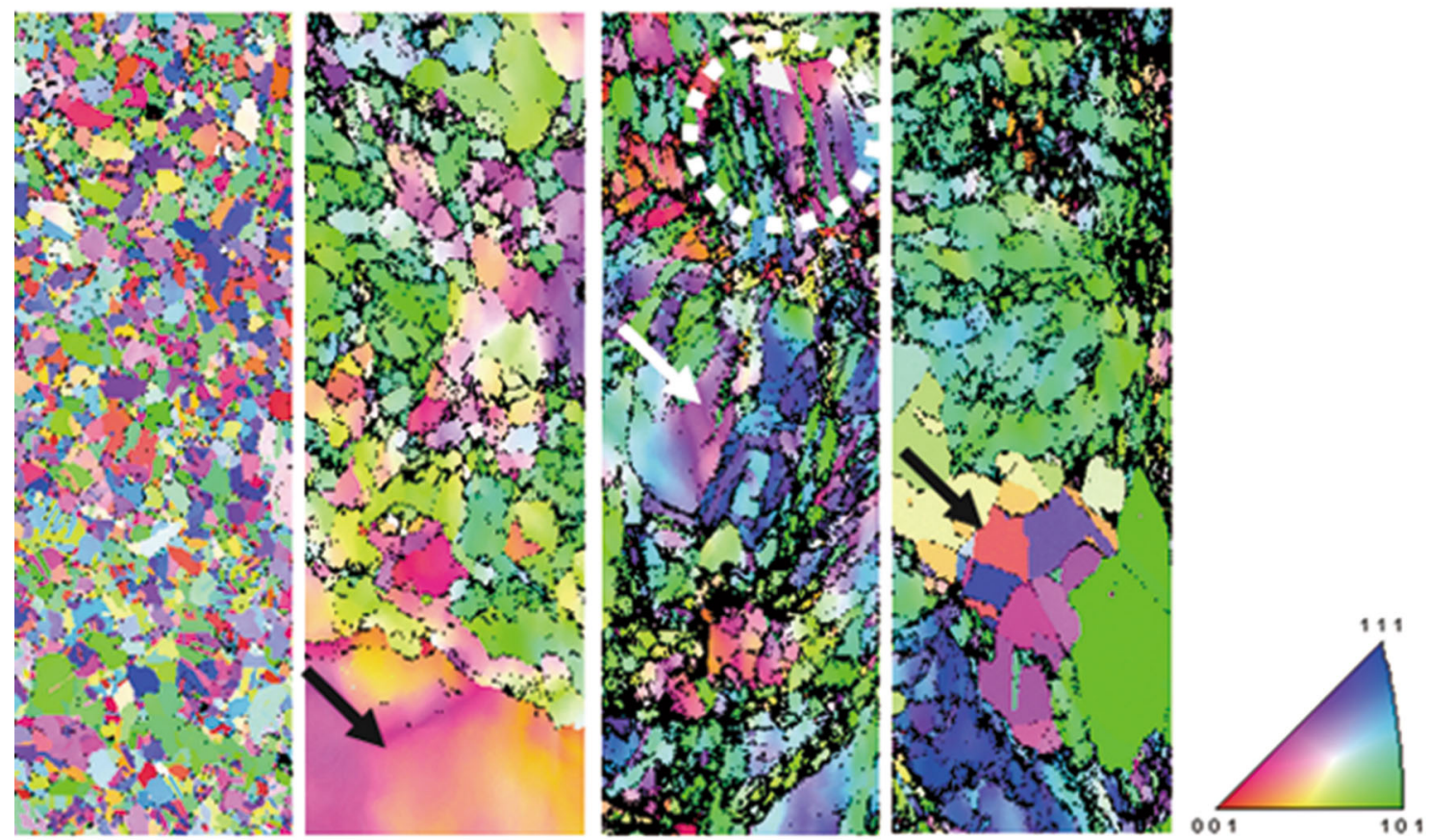

Grain Orientation Spread (GOS) maps

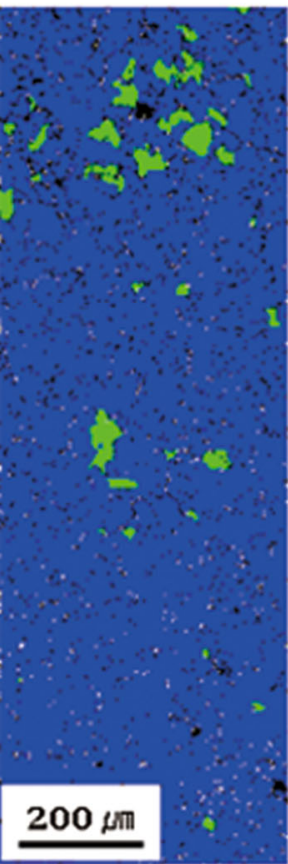

Initial

(a)

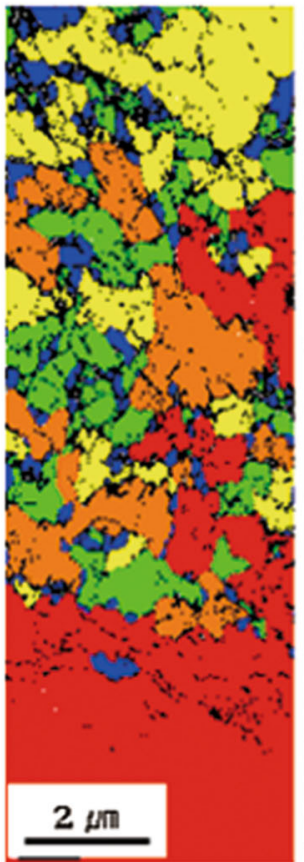

RTD sample

(b)

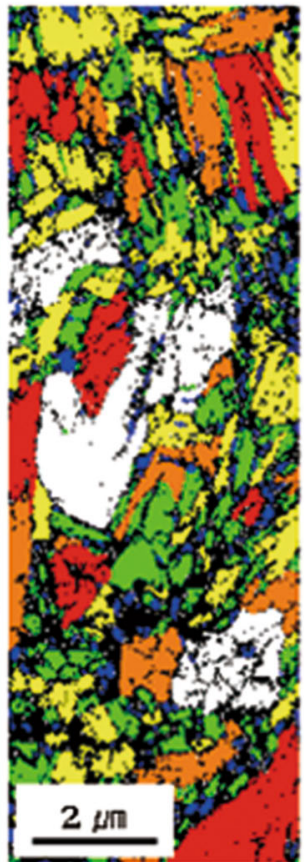

CTD sample 1

(c)

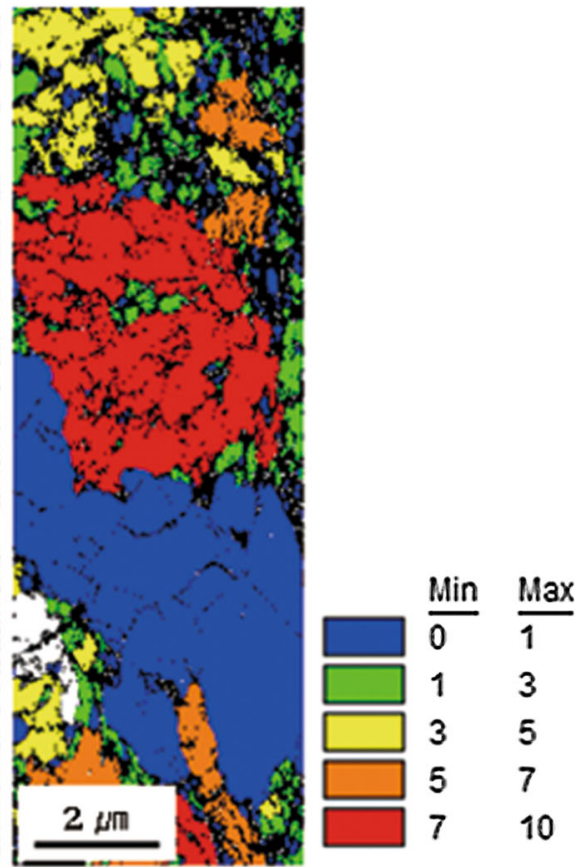

CTD sample 2

(d)

Fig. 5-EBSD results of $(a)$ the initial and the deformed specimens in the $(b)$ RTD sample, $(c)$ CTD sample 1, and (d) CTD sample 2: inverse pole figure (IPF) and grain orientation spread (GOS) maps.

Larger strain (i.e., higher energy) decreases the required temperature and time for activating migration recrystallization. Therefore, an SPD-processed sample with very fine grains (high surface energy) and high defect densities (high strain energy) has low thermal stability. ${ }^{[27-29]}$ The CTD sample was also in a very high-energy state due to its relatively large strain, high strain rate, and very low processing temperature. Consequently, it is inferred that the temperature condition for partial recrystallization decreases to include room temperature. 


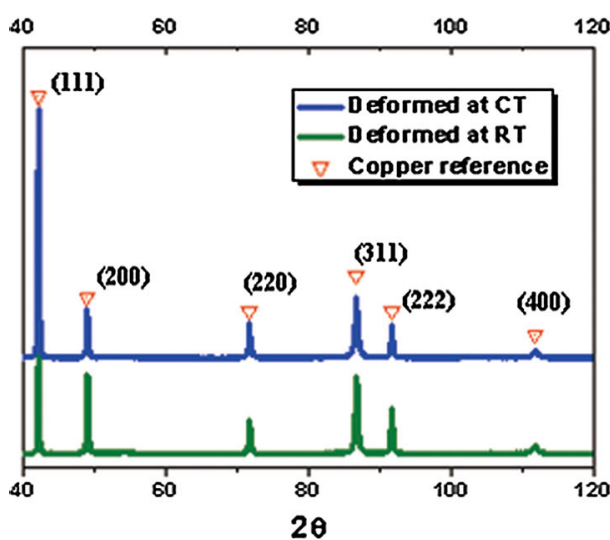

(a)
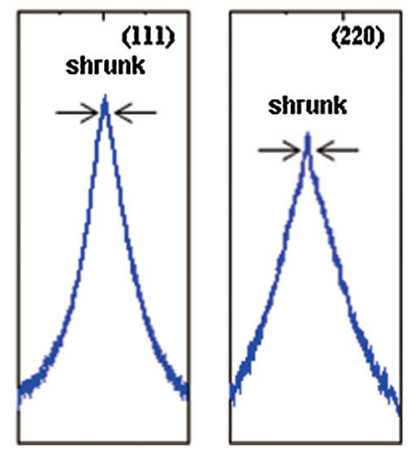

(c)

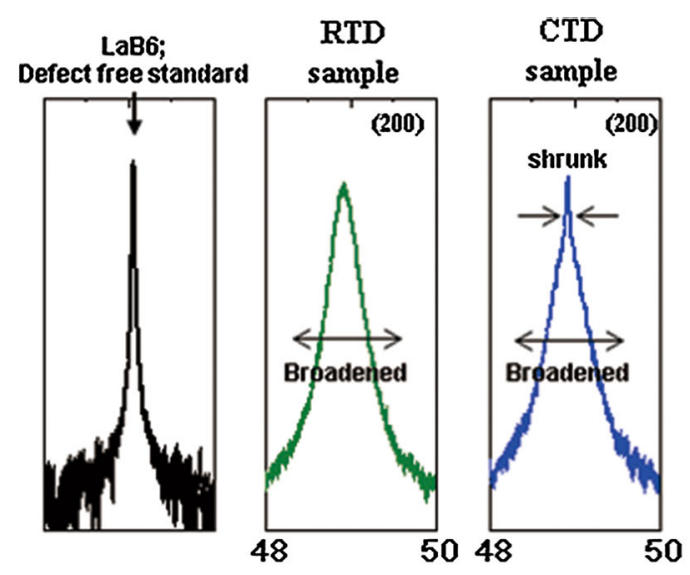

(b)

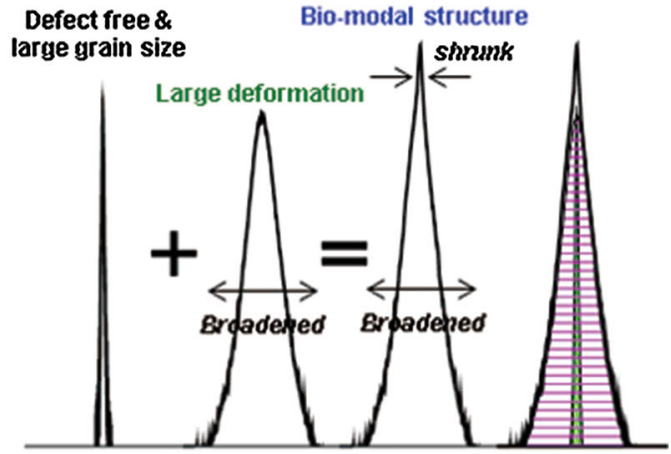

(d)

Fig. 6-Synchrotron XRD peak analyses: (a) whole peaks of the RTD and CTD samples and reference points of pure copper, $(b)$ one of the reference peaks from the standard LaB6 sample, and the (200) peaks from the RTD and CTD samples in logarithmic scale, $(c)$ the other peaks of (111), (220), and (311) of the CTD sample in logarithmic scale, and (d) schematic combination of peaks from bi-modal structure.

Deformation of copper at cryogenic temperature with large strain is not the only requirement for micro/ ultrafine bi-modal structure. Edalati et al.$^{[10]}$ presented a very unstable microstructure for both high purity (99.99999 pct) and commercial purity (99.99 pct) copper, which finally became a fully recrystallized microstructure at room temperature, 200 minutes after the cryogenic HPT process. Contrary to this case, relatively stable microstructure was maintained in our CTD sample. Two possible reasons exist for such a difference in microstructure. First is lower vacancy density in the CTD sample, related to diffusion affecting microstructure evolution, ${ }^{[30]}$ due to relatively lower pressure and strain than in samples after the HPT process. ${ }^{[31]}$ The second involves relatively stable twin boundaries generated during deformation under conditions of cryogenic temperature, high strain rate, and moderate strain. ${ }^{[10,32]}$ A proper amount of strain $(\sim 1.7)$ and stable twin boundaries, which maintain tolerable misorientations, also suppress the activation of full recrystallization during diffusion-related recrystallization.

Twin boundaries are more critical for microstructural refinement and defect stability than other defects. ${ }^{[33,34]}$ These two features enable twin boundaries to exist (even at high temperature) after deformation and enable the materials to have great strength. ${ }^{[6]}$ High speed and low temperature (over $\sim 33$ of $\ln Z$ ) are required for twinning to be activated as one of the main deformation mechanisms in copper, and the twin fraction increases with increasing $\ln Z \cdot^{[7,8]}$ Thus, the CTD sample with $\sim 64$ of $\ln Z$ could have many twin boundaries. However, the whole microstructure was not covered by twins due to the limitation of the volume fraction of the deformation twins. This heterogeneously deformed microstructure indicates a non-uniform energy state in the microstructure of the CTD sample. Therefore, some regions of the samples having high-energy states could be recrystallized after warming to room temperature from cryogenic temperature.

The bi-modal structure induced by warming a cryogenically cold sample to room temperature is hard to predict. This is particularly true when considering only the value of $\ln Z$, which represents the characteristics of the microstructure after deformation under a specific condition. As in the CTD sample case, it should be kept in mind when designing microstructure by deformations, that the change in temperature after deformation is a key factor, determining the microstructure. By controlling the volume-fraction of twinning and the deformation temperature, unusual structure, such as bi-modal, could be produced in the CTD sample. Moreover, such microstructure could have a potential 
for achieving attractive properties. For example, it has been reported that both high strength and ductility could be obtained by bi-modal structure. ${ }^{[14]}$

\section{CONCLUSIONS}

Pure copper was deformed at a high strain rate at the temperature of liquid nitrogen. The bi-modal microstructure, a mixture of ultrafine and micro-grained structures, was identified locally by EBSD observations and globally by XRD measurements. Despite the presence of inhomogeneous microstructure, uniform and extreme micro-hardness was obtained. The partial recrystallization leading to bi-modal features was caused by the inhomogeneous energy state of the microstructure and its thermal stability. Deformation twinning is considered as one of the main reasons for the partial recrystallization after the sample warmed to room temperature. Although further investigation is required, the present results demonstrate the potential of cryogenic deformation at strain rates over $10^{3} \mathrm{~s}^{-1}$, as a new approach for designing and producing materials with bi-modal microstructure.

\section{ACKNOWLEDGMENT}

This study was supported by A.D.D. through basic research Project No. 11-01-04-08. This work was supported by the National Research Foundation of Korea (NRF) Grant funded by the Korean Government (MSIP) (No. 2014R1A2A1A10051322).

\section{REFERENCES}

1. A.P. Zhilyaev and T.G. Langdon: Prog. Mater Sci., 2008, vol. 53, pp. 893-979.

2. R.Z. Valiev and T.G. Langdon: Prog. Mater Sci., 2006, vol. 51, pp. 881-981.

3. K. Hajizadeh and B. Eghbali: Met. Mater. Int., 2014, vol. 20, pp. $343-50$.

4. Y. Wang, H. Yang, C. Zhang, and F. Yu: Met. Mater. Int., 2015, vol. 21 , pp. 260-69.

5. H.S. Kim, S.-H. Joo, and H.J. Jeong: Korean J. Met. Mater., 2014, vol. 52, pp. 87-99.
6. B. Zhang and V.P.W. Shim: Acta Mater., 2010, vol. 58, pp. $6810-827$.

7. T.L. Brown, C. Saldana, T.G. Murthy, J.B. Mann, Y. Guo, L.F. Allard, A.H. King, W.D. Compton, K.P. Trumble, and S. Chandrasekar: Acta Mater., 2009, vol. 57, pp. 5491-5500.

8. Y.S. Li, Y. Zhang, N.R. Tao, and K. Lu: Acta Mater., 2009, vol. 57, pp. 761-72.

9. Y.S. Li, N.R. Tao, and K. Lu: Acta Mater., 2008, vol. 56, pp. $230-41$.

10. K. Edalati, J.M. Cubero-Sesin, A. Alhamidi, I.F. Mohamed, and Z. Horita: Mat. Sci. Eng. A, 2014, vol. 613, pp. 103-10.

11. B. Banerjee: arXiv preprint cond-mat $/ 0512466,2005$.

12. H.S. Kim: Mater. Trans., 2001, vol. 42, pp. 536-38.

13. T. Suo, Y.S. Li, F. Zhao, X. Fan, and W. Guo: Mech. Mater., 2013, vol. 61, pp. 1-10.

14. Y.M. Wang and E. Ma: Acta Mater., 2004, vol. 52, pp. 1699-1709.

15. D. Gomez-Garcia, B. Devincre, and L.P. Kubin: Phys. Rev. Lett., 2006, vol. 96, p. 125503.

16. G.J. Fan, L.F. Fu, H. Choo, P.K. Liaw, and N.D. Browning: Acta Mater., 2006, vol. 54, pp. 4781-92.

17. G.J. Fan, Y.D. Wang, L.F. Fu, H. Choo, P.K. Liaw, Y. Ren, and N.D. Browning: Appl. Phys. Lett., 2006, vol. 88, p. 171914.

18. U. Andrade, M.A. Meyers, K.S. Vecchio, and A.H. Chokshi: Acta Metall. Mater., 1994, vol. 42, pp. 3183-95.

19. J. Ćížek, I. Procházka, M. Cieslar, R. Kužel, J. Kuriplach, F. Chmelík, I. Stulíková, F. Bečváŕ, O. Melikhova, and R.K. Islamgaliev: Phys. Rev. B, 2002, vol. 65, p. 094106.

20. W.H. Huang, C.Y. Yu, P.W. Kao, and C.P. Chang: Mat. Sci. Eng. A, 2004, vol. 366, pp. 221-28.

21. N. Tsuji, R. Ueji, Y. Minamino, and Y. Saito: Scripta Mater., 2002, vol. 46, pp. 305-10.

22. X. Molodova, G. Gottstein, M. Winning, and R.J. Hellmig: Mat. Sci. Eng. A, 2007, vol. 460, pp. 204-13.

23. Ai. Zi: Mater. Charact., 2010, vol. 61, pp. 141-44.

24. T.S. Wang, F.C. Zhang, M. Zhang, and B. Lv: Mat. Sci. Eng. A, 2008, vol. 485, pp. 456-60.

25. H. Azizi-Alizamini, M. Militzer, and W.J. Poole: Scripta Mater., 2007, vol. 57, pp. 1065-68.

26. Y.D. Wang, M. Chen, F. Zhou, and E. Ma: Nature, 2002, vol. 419, pp. $912-15$.

27. C.F. Gu and C.H.J. Davies: Mat. Sci. Eng. A, 2010, vol. 527, pp. 1791-99.

28. H. Jiang, Y.T. Zhu, D.P. Butt, I.V. Alexandrov, and T.C. Lowe: Mat. Sci. Eng. A, 2000, vol. 290, pp. 128-38.

29. A.V. Stolbovsky, V.V. Popov, E.N. Popova, and V.P. Pilyugin: Bull. Russ. Acad. Sci. Phys., 2014, vol. 78, pp. 908-16.

30. R.Z. Valiev, Y.V. Ivanisenko, E.F. Rauch, and B. Baudelet: Acta Mater., 1996, vol. 44, pp. 4705-12.

31. D. Setman, E. Schafler, E. Korznikova, and M.J. Zehetbauer: Mat. Sci. Eng. A, 2008, vol. 493, pp. 116-22.

32. R.L. Fullman: J. Appl. Phys., 1951, vol. 22, pp. 448-55.

33. C. Saldana, A.H. King, E.A. Stach, W.D. Compton, and S. Chandrasekar: Appl. Phys. Lett., 2011, vol. 99, p. 231911.

34. C. Ye, S. Suslov, D. Lin, Y. Liao, X. Fei, and G.J. Cheng: J. Appl. Phys., 2011, vol. 110, p. 083504. 